\title{
A Possible Mechanism of NK Cell-lineage Granular Lymphocyte Proliferative Disorder (NK-GLPD) in a Patient with Chronic Active Epstein-Barr Virus Infection (CAEBV) and Severe Hypersensitivity to Mosquito Bites (SHMB)
}

\author{
Shiro Ohshima, Masaru Ishi, Hideo AsadA*, Toyoshi TAtekawA**, Norihiko Yamaguchi, \\ Hideyuki Kobayashi, Taeko IshII, Toru Mima, Ichiro KaWASE and Yukihiko SAEKI
}

\begin{abstract}
We report the case of a young female patient with chronic active Epstein-Barr virus infection (CAEBV) and severe hypersensitivity to mosquito bites (SHMB). She showed a marked increase of NK cell population in peripheral blood. The NK cell population was suggested to be infected with EBV, and to be oligoclonal by Southern blotting using an EBV genome terminal-repeat probe. The NK cells aberrantly expressed CD25, a high affinity receptor for IL-2, and showed an augmented in vitro proliferative response to IL-2. Moreover, they also showed enhanced expression of both Fas-ligand and Bcl-2, and resistance to in vitro Fasinduced apoptotic cell death (Fas-ACD). Taken together, these observations suggested that both the augmentation of proliferative response to IL-2 and the decrease in FasACD may cause NK cell lineage granular lymphocyte proliferative disorder (NK-GLPD) in patients with CAEBV and SHMB.
\end{abstract}

(Internal Medicine 41: 651-656, 2002)

Key words: CD25, IL-2, Fas, Fas ligand

\section{Introduction}

Severe hypersensitivity to mosquito bites (SHMB) is a rare disease and most patients are Asian, including Japanese. It is characterized clinically by intense skin reactions at mosquito bite sites with systemic symptoms (1). The initial skin lesion presents typically with erythema, swelling, and a bulla that subsequently develops into necrosis or an ulcer, and is usually accompanied by high grade fever as a systemic symptom. Another important clinical feature is the high mortality rate due to complications, such as malignant NK cell-large granular lymphoproliferative disorder (NK-GLPD) and hemophagocytic syndrome (HPS) (2). Recent studies have shown that chronic active Epstein-Barr virus infection (CAEBV) is closely associated with SHMB and its malignant complications (3-5). In addition, NK cell expansion in the periphery, referred to as NK-GLPD, is often observed $(4,6)$. Thus, in conjunction with the high incidence of malignant NK-GLPD, the expanded oligoclonal NK cells of patients with SHMB and CAEBV may represent a preneoplastic population of NK cells. Therefore, clarification of the mechanism of NK-GLPD in patients with SHMB and CAEBV will provide important information on the oncogenesis of NK cells.

For editorial comment, see p 603.

\section{Case Report}

The patient was an 18-year-old woman. Since the summer of 1994, when she was 14 years old, she had intense skin reactions at mosquito bite sites. The skin lesions usually developed into ulcers and healed with scarring. In addition to such local cutaneous reactions, she also manifested systemic symptoms such as high fever and general malaise. Thus, she was diagnosed as having SHMB. At that time, she was found to have an abnormal elevation of serum antibodies to EBV.

In 1998, she was admitted to our hospital for further examination. On admission, her body temperature was normal at

From the Departments of Molecular Medicine, *Dermatology, Osaka University Medical School, Suita and **Division of Internal Medicine, Kinki-Central Hospital, Itami

Received for publication August 6, 2001; Accepted for publication March 27, 2002

Reprint requests should be addressed to Dr. Yukihiko Saeki, the Department of Molecular Medicine, Osaka University Medical School, 2-2 Yamada-oka, Suita, Osaka 565-0871 


\section{OHshima et al}

$36.6^{\circ} \mathrm{C}$. With the exception of multiple intense hemorrhagic necrotic skin lesions and scars in addition to oral aphtha, no other remarkable physiological findings were noted. As shown in Table 1, laboratory examinations revealed a red blood cell count of $428 \times 10^{4} / \mathrm{mm}^{3}$, hemoglobin level of $12.2 \mathrm{~g} / \mathrm{dl}$, white blood cell count of $4,670 / \mathrm{mm}^{3}$, platelet count of $23.3 \times 10^{4} / \mathrm{mm}^{3}$, potassium level of $4.1 \mathrm{mEq} / l$, sodium level of $138 \mathrm{mEq} / l$, urea nitrogen level of $11 \mathrm{mg} / \mathrm{dl}$, creatinine level of $0.6 \mathrm{mg} / \mathrm{dl}$. The level of serum C-reactive protein was not elevated $(<0.2 \mathrm{mg} /$ dl). Glucose, liver enzymes, and bilirubin were normal. Although the white blood cell count was normal, the percentage of lymphocytes was markedly increased to $54.4 \%$. On immunological examinations, the serum immunoglobulin levels were IgG of $1,813 \mathrm{mg} / \mathrm{dl}, \mathrm{IgA}$ of $389 \mathrm{mg} / \mathrm{dl}$, and IgM of $112 \mathrm{mg} / \mathrm{dl}$. Serum IgE level was markedly increased to $10,000 \mathrm{IU} / \mathrm{ml}$. Neither rheumatoid factor nor anti-nuclear antibodies was present. The serum complement levels were $\mathrm{C}_{3}$ of $97 \mathrm{mg} / \mathrm{dl}$ and $\mathrm{C}_{4}$ of $22 \mathrm{mg} / \mathrm{dl}$. Abdominal ultrasonography revealed moderate hepatosplenomegaly. However, her bone marrow smear showed normocellularity with the absence of morphological abnormalities and phagocytosis. The pattern of serum EBVspecific antibodies was compatible with chronic active EBV infection (CAEBV) as the anti-EBVCA IgG level was mark- edly increased $(\times 2,560)$, whereas the anti-EBNA level was not increased $(\times 80)$. Flow cytometric analysis showed a marked increase in the CD56+, CD3-population, which was suggested to be NK cells, at $51.1 \%$.

We have observed no signs of malignancy to date.

\section{Materials and Methods}

The following experiments were performed with both the informed consent of the patient and the approval of the Osaka University Human Research Committee.

\section{Purification of NK cells and their characterization}

Peripheral blood mononuclear cells were isolated by density-gradient centrifugation. The NK cells were purified by magnetic cell sorting (MACS) using a StemSep ${ }^{\mathrm{TM}}$ kit (Stem Cell Technologies Inc., British Columbia, Canada) as described elsewhere (7). The phenotypes of purified cells were examined by flow cytometry using antibodies to CD3, CD16, and CD56. After MACS, more than $99 \%$ of the collected cells were CD3-, CD16+, CD56+; i.e. the typical NK cell phenotype. In addition, these cells showed the typical morphology of large granular lymphocytes (LGL) (Fig. 1). All of the fol-

Table 1. Laboratory Data on Admission

\begin{tabular}{lrcr}
\hline Peripheral blood examination & & Immunological examination & \\
White blood cells & $4,670 / \mathrm{mm}^{3}$ & Immunoglobulin G & $1,813 \mathrm{mg} / \mathrm{dl}$ \\
Neutrophils & $39.2 \%$ & Immunoglobulin A & $389 \mathrm{mg} / \mathrm{dl}$ \\
Lymphocytes & $54.4 \%$ & Immunoglobulin M & $112 \mathrm{mg} / \mathrm{dl}$ \\
CD3+ & $(42.2 \%)$ & Immunoglobulin E & $10,000 \mathrm{IU} / \mathrm{ml}$ \\
CD19+ & $(11.2 \%)$ & Anti-nuclear antibody & $<\times 40$ \\
CD4+ & $(26.6 \%)$ & Rheumatoid factor & $<10 \mathrm{IU} / \mathrm{ml}$ \\
CD8+ & $(13.5 \%)$ & Complement 3 & $97 \mathrm{mg} / \mathrm{dl}$ \\
CD56+ & $(51.1 \%)$ & Complement 4 & $22 \mathrm{mg} / \mathrm{dl}$ \\
Eosinophils & $2.4 \%$ & & \\
Basophils & $0.4 \%$ & EBV-specific antibodies & \\
Monocytes & $3.6 \%$ & EB-VCA IgG & $\times 2,560$ \\
Red blood cells & $428 \times 10^{4} / \mathrm{mm}^{3}$ & EB-VCA IgM & - \\
Hemoglobin & $12.2 \mathrm{~g} / \mathrm{dl}$ & EB-VCA IgA & $\times 160$ \\
Hematocrit & $37.6 \%$ & EB-EA IgG & $\times 20$ \\
Platelets & $23.3 \times 10^{4} / \mathrm{mm}$ & EB-EA IgM & $\times 80$ \\
& & EB-EBNA & \\
Chemical analysis & & & \\
& & & \\
C-reactive protein & $<0.2 \mathrm{mg} / \mathrm{dl}$ & & \\
Sodium & $138 \mathrm{mEq} / l$ & & \\
Potassium & $4.1 \mathrm{mEq} / l$ & & \\
Blood urea nitrogen & $11 \mathrm{mg} / \mathrm{dl}$ & & \\
Creatinine & $0.6 \mathrm{mg} / \mathrm{dl}$ & & \\
Aspartate aminotransferase & $17 \mathrm{IU} / l$ & & \\
Alanine aminotransferase & $10 \mathrm{IU} / l$ & & \\
$\gamma$-Glutamyltransferase & $14 \mathrm{IU} / l$ & & \\
Lactate dehydrogenase & $238 \mathrm{IU} / l$ & & \\
Total protein & $8.2 \mathrm{~g} / \mathrm{dl}$ & & \\
Albumin & $4.2 \mathrm{~g} / \mathrm{dl}$ & & \\
\hline
\end{tabular}




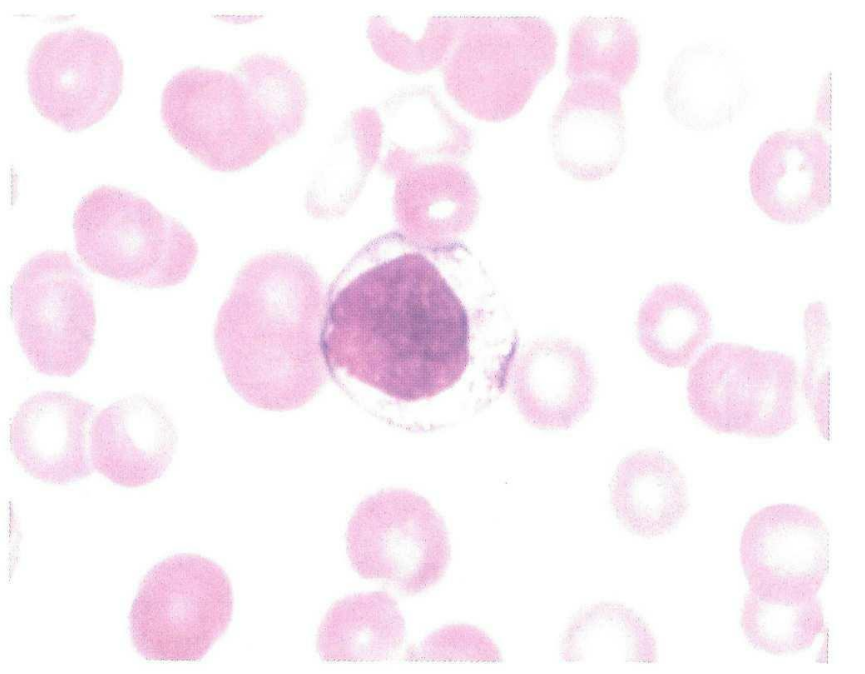

Figure 1. LGL morphology observed in the patient. MayGruenwald-Giemsa staining shows the morphology of large granular lymphocyte (LGL) with azurophilic granules and abundant cytoplasm under light microscopy $(\times 1,000)$.

lowing experiments were performed using the highly purified NK cells obtained by MACS. Further characterization of the highly purified NK cells from the patient (P-NK cells) was performed by flow cytometry using antibodies to $\mathrm{CD} 25$, Fas, Fas L and Bcl-2.

\section{Detection of EBV}

The detection of EBV in P-NK cells was performed both by in situ hybridization assay for EBV-encoded small nuclear RNAs 1 (EBER 1) (8) and by PCR for EBV DNA (9).

\section{Clonality of P-NK cells}

The clonality of P-NK cells was assessed by Southern blot analysis using a probe for the terminal repeat of EBV as described previously (10).

Cell culture and assessment of proliferative response to IL-2

Highly purified NK cells $\left(5 \times 10^{5}\right.$ cells/well) were cultured in the absence or presence of different doses of IL-2. Cell proliferation was quantified by $\left[{ }^{3} \mathrm{H}\right]$-thymidine incorporation on day 3. All experiments were performed in triplicate culture.

\section{Results}

First, to examine whether the expanded NK cells were infected with EBV, the presence of EBV was assessed both by in situ hybridization assay for EBV-encoded small nuclear RNA 1 (EBER 1) and by PCR for EBV DNA. As shown in Fig. 2, the in situ hybridization analysis showed that more than $90 \%$ of NK cells from the patient (P-NK cells) were positive for EBER 1. In addition, in the PCR study for EBV, a distinct band was observed in the lane of P-NK cells at the same position as

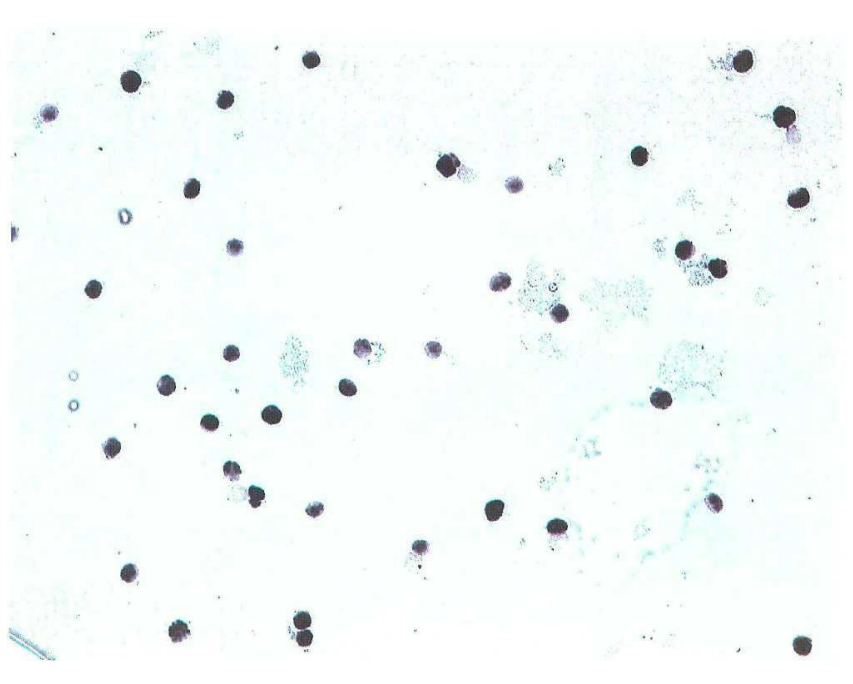

Figure 2. Detection of EBER 1 expression in P-NK by in situ hybridization. In situ hybridization was performed in P-NK using the DIG-labeled antisense EBER 1 oligoprobe. Strong signals, indicated as stained cells, were observed in $90 \%$ of cytospinned P-NK $(\times 200)$.

Table 2. Expression of EBV Latent Proteins in P-NK Cells

\begin{tabular}{cccccc}
\hline EBER & EBNA1 & EBNA2 & LMP1 & LMP2A & LMP2B \\
\hline+ & + & - & + & - & -
\end{tabular}

EBER: EBV-encoded small RNA, EBNA: EBV nuclear antigen, LMP: latent membrane protein. Total RNA was extracted from P-NK celles using the guanidine isothiocyanate-phenol method. The first strand cDNA was synthesized from $5 \mathrm{mg}$ of total RNA using the Superscript preamplification system (Life Technologies). RT-PCR was performed using primers specific for each latent gene as described previously (20).

the positive control, while no bands were observed in the lane of NK cells from the healthy control (C-NK cells) (data not shown). These findings suggested that P-NK cells were infected with EBV. Moreover, Southern hybridization assay using the terminal repeat probe $(10)$ showed that P-NK cells were oligoclonal (data not shown). In addition, RT-PCR analysis for the expression of EBV latent proteins and replication-associated proteins showed that P-NK cells expressed EBER 1, EBV nuclear antigen 1 (EBNA 1), and latent membrane protein 1 (LMP 1), whereas neither EBNA 2 nor LMP 2 was expressed. This EBV gene expression pattern of P-NK is referred to as latency II (Table 2).

Next, we examined expression of CD25, a high affinity receptor for IL-2, by flow cytometry. P-NK cells showed significant $\mathrm{CD} 25$ expression (Fig. 3 ). In addition, P-NK cells showed an enhanced proliferative response to IL-2 (Fig. 4). Moreover, flow cytometry also showed enhanced expression of both Fas 

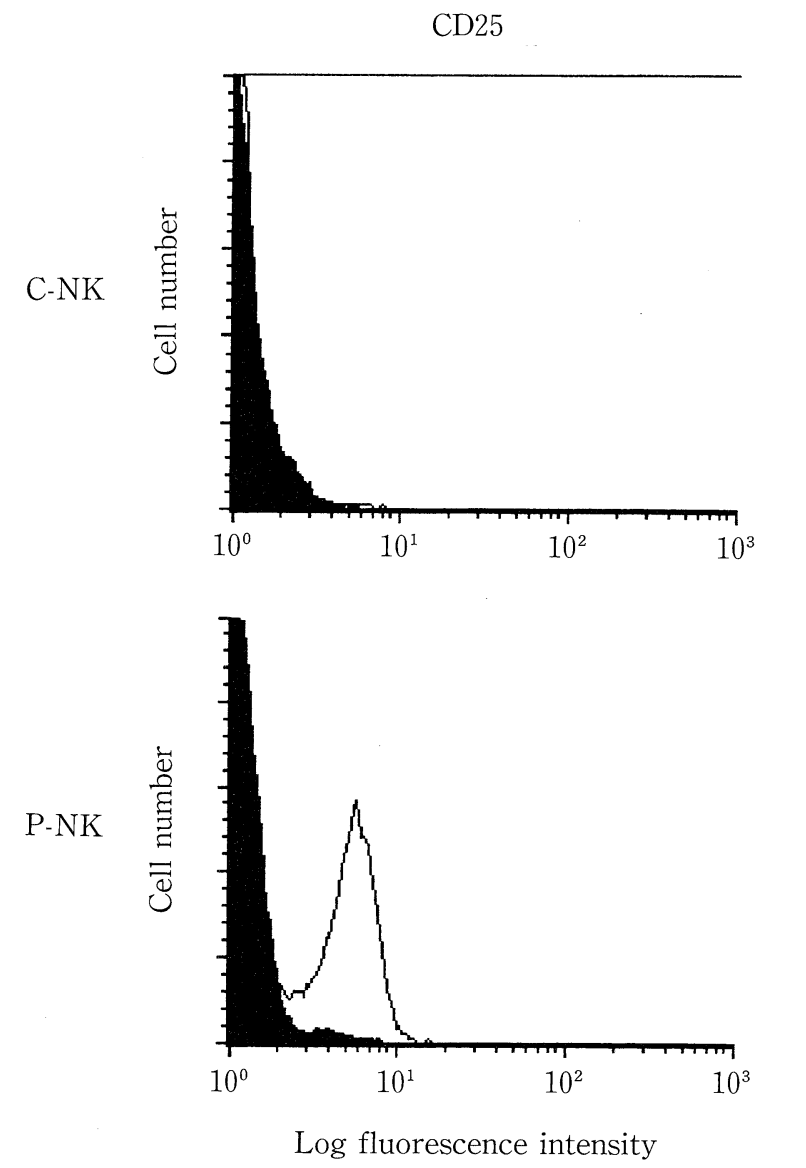

Figure 3. Flow cytometric analysis of CD25 on P-NK and CNK. The expressions of CD25 on P-NK and C-NK were investigated by staining with FITC-conjugated anti-CD25 antibodies. Upper figure shows C-NK stained with FITC-conjugated antiCD25 antibodies (solid line) and with FITC-conjugated control antibodies (shaded area). Lower figure shows P-NK stained with FITC-conjugated anti-CD25 antibodies (solid line) and with FITC-conjugated control antibodies (shaded area). CD25 was expressed on P-NK, whereas never on C-NK.

ligand (Fas L) and Bcl-2 in comparison with C-NK cells (Fig. 5). Although P-NK cells showed significant expression of Fas, anti-Fas antibody, $\mathrm{CH}$ 11, did not induce apoptosis in these cells (data not shown). In addition, soluble Fas L levels were significantly elevated in both the culture supernatant of P-NK cells and in the patient's serum (Table 3 ).

\section{Discussion}

In this study, we demonstrated several interesting findings using highly purified NK cells from a patient with CAEBV and SHMB (P-NK cells). First, the P-NK cells were infected with EBV according to the findings of both in situ hybridization for EBER 1 and PCR for EBV DNA. However, CD21 (CR2), a well-known receptor for EBV, was not expressed on

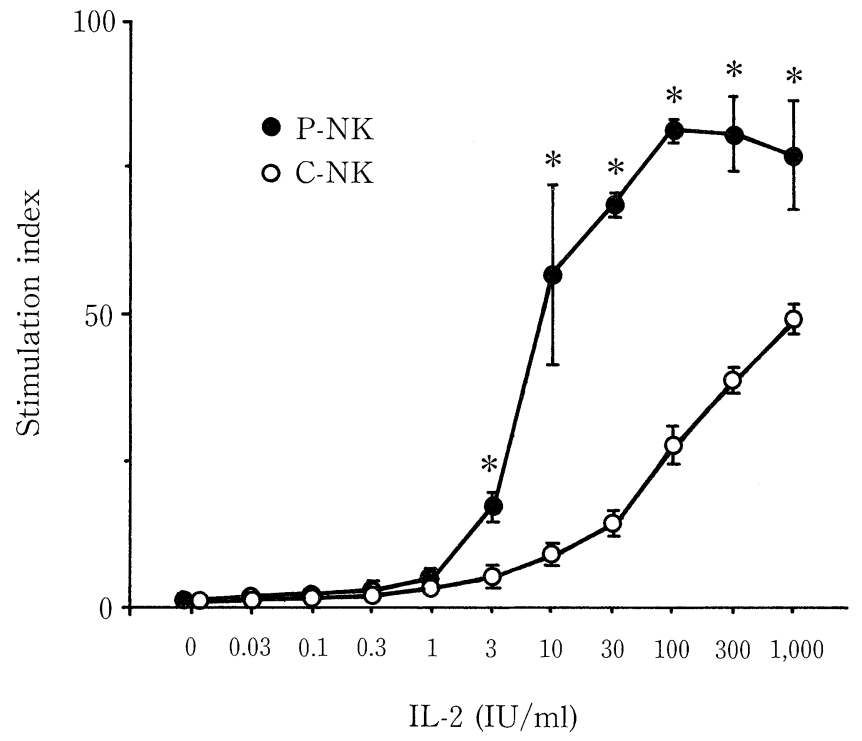

Figure 4. The proliferative response to IL-2 in P-NK and C-NK. Either P-NK or C-NK $\left(5 \times 10^{4}\right.$ cells/well $)$ were cultured with 0.2 ml of complete culture medium supplemented with $10 \%$ FCS in the presence of indicating dose $(0-1,000 \mathrm{IU} / \mathrm{ml})$ of recombinant IL-2 in 96-well plates for 72 hours. Cell proliferative response was measured by $\left[{ }^{3} \mathrm{H}\right]$ thymidine incorporation. Closed circles indicate the proliferative responses to IL-2 in P-NK, and opened circles indicated those in C-NK. Although IL-2 dependent proliferative response was observed in either $\mathrm{P}-\mathrm{NK}$ or $\mathrm{C}-\mathrm{NK}$, the response rate in $\mathrm{P}$-NK was significantly higher than that in $\mathrm{C}$ NK $(* \mathbf{p}<0.001)$.

P-NK cells (data not shown). Although the mechanism by which EBV infected these NK cells initially remains unknown, it is not surprising because previous reports have demonstrated that EBV can infect not only B cells but also other lymphocytes including $\mathrm{T}$ cells and NK cells $(11,12)$. Moreover, the findings of Southern hybridization using an EBV terminal repeat probe suggested that P-NK cells were oligoclonal. The expression pattern of EBV latent proteins and replication-associated proteins of P-NK suggested the latency pattern of EBV that was referred to as latency II. This pattern of EBV latency is well known to be associated with EBV-related malignancies such as nasopharyngeal carcinoma (13), EBV-positive T cell lymphoma (14), and Hodgkin's disease (15). As not only EBNA 1 but also other EBV latent proteins, such as LMP, can serve as target antigens for EBV-specific cytotoxic T cells, P-NK cells may still be sensitive to EBV-specific cytotoxic T cells. However, the reduced expression of EBNAs in P-NK cells suggested that the EBV-specific cytotoxic T cell response against P-NK cells was reduced.

Second, CD25, a high affinity IL-2 receptor, was highly expressed on P-NK cells. This is unique for P-NK cells because NK cells usually express only a low affinity IL-2 receptor, composed of $\beta$ and $\gamma$ chains, and not the high affinity receptor, CD25. In fact, P-NK cells showed an enhanced prolif- 

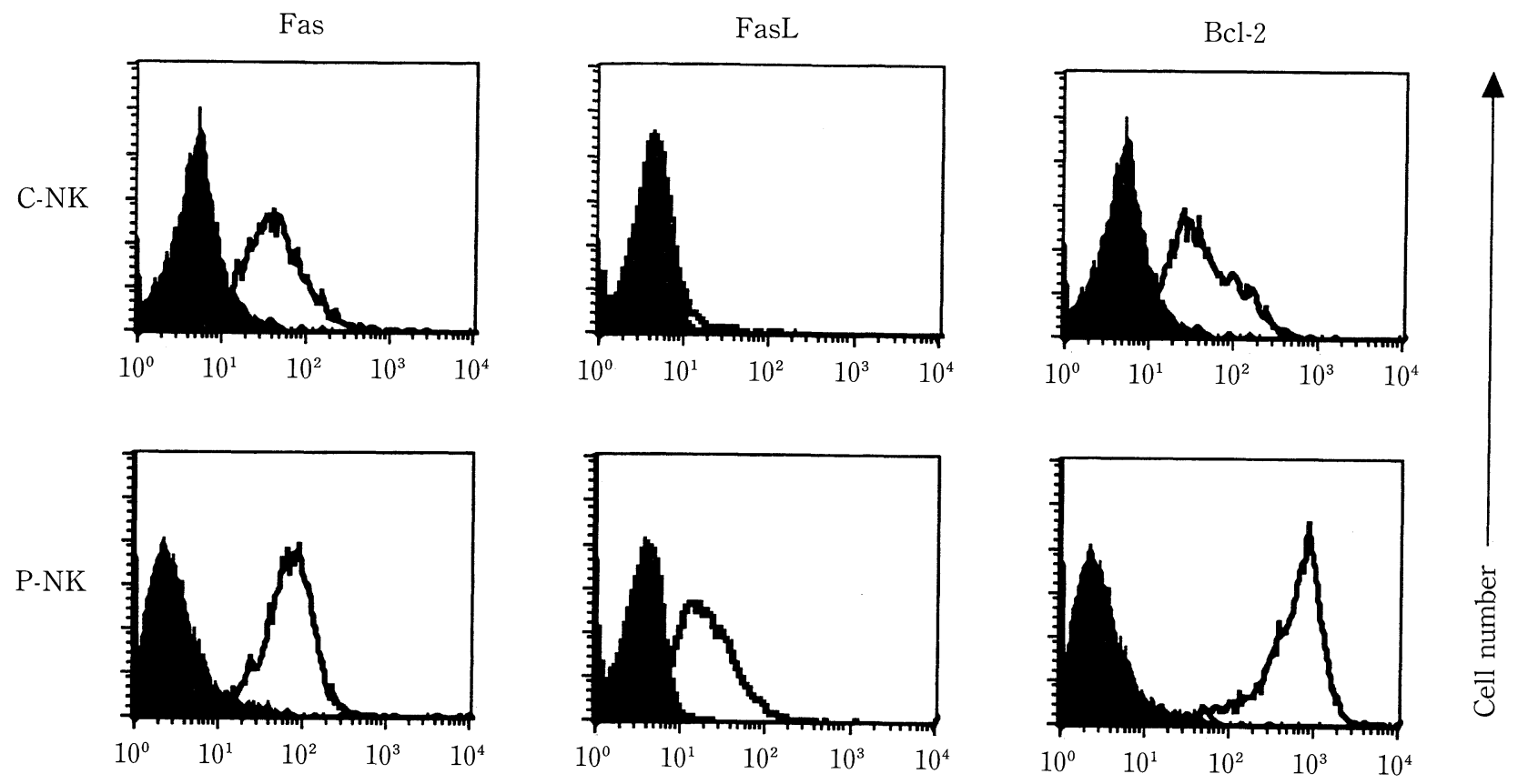

Log fluorescence intensity

Figure 5. The expression of Fas, FasL, and Bcl-2 on P-NK and C-NK. The expressions of Fas (left panels), FasL (middle panels) and Bcl-2 (right panels) on either C-NK (three upper panels) or P-NK (three lower panels) or were also investigated by staining with FITC-conjugated anti-Fas, biotinylated anti-Fas ligand (clone 4H9) and anti-Bcl-2 antibodies. As for either Fas or Bcl-2, either C-NK (upper figures) or P-NK (lower figures) stained with FITC-conjugated antibodies specific for either Fas or Bcl-2 (solid line) and with FITC-conjugated control antibodies (shaded area). As for Fas ligand, the cells were preincubated at $37^{\circ} \mathrm{C}$ for 48 hours in the presence of $10 \mu \mathrm{M}$ of BB-2116 (matrix metalloproteinase inhibitor). Subsequently, the cells were stained with biotinylated anti-Fas ligand in the absence (solid line) or presence (shaded area) of $5 \mu \mathrm{g} / \mathrm{ml}$ of recombinant Fas ligand.

Table 3. The Concentration of $\mathrm{sFas} \mathrm{L}$ in Serum and Culture Supernatant

\begin{tabular}{lccc}
\hline & Serum & Culture supernatant & \\
\hline Patient & 698 & 3,634 & \\
Control & $<50$ & $<50$ & $(\mathrm{pg} / \mathrm{ml})$ \\
\hline
\end{tabular}

The concentrations of soluble Fas ligand (sFas L) in the serum and the culture supernatant of P-NK cells (Patient) or C-NK cells (Control) were measured using ELISA as described previously (21).

erative response to IL-2 in vitro compared to C-NK cells. A similar observation has been recently reported by Tsuge et al (6). This may have contributed to the abnormal expansion of NK cells in this patient. Although the reason remains unknown, it is suggested that the CAEBV (chronic active EBV infection) might be associated with the aberrant expression of CD25 on P-NK cells, as Kube et al demonstrated that EBNA1 induced CD25 in a Hodgkin cell line (16). In addition, Kawa-Ha et al pointed out the risk of IL-2 administration in the treatment of

\section{NK-GLPD (17).}

Third, P-NK cells were resistant to Fas-induced apoptotic cell death (Fas ACD). There was no difference in Fas expression between P-NK cells and C-NK cells. However, P-NK cells showed enhanced expression of Bcl-2 compared with C-NK cells. This was not surprising because P-NK cells expressed LMP1, which can induce Bcl-2 (18). As Bcl-2 is well known to be an anti-apoptotic molecule, the enhanced expression of Bcl-2 may have caused the decrease in Fas ACD in P-NK cells.

Finally, P-NK cells overexpressed Fas $L$ and secreted soluble Fas L (sFas L). In addition, the serum sFas L level of the patient was significantly elevated compared with that of the healthy controls. It is well known that T cells, including cytotoxic $\mathrm{T}$ cells, constitutively express Fas on the cell surface. Therefore, P-NK cells expressing Fas L on the cell surface and secreting sFas L, might evade the attack by EBV-specific cytotoxic $T$ cells through both surface Fas $L$ and secreted sFas $L$, as reported in the tumor system (19). Moreover, overexpression of the membrane form of Fas $\mathrm{L}$ and enhanced secretion of $\mathrm{sFas}$ L by P-NK cells might be related to organ (or tissue) damage, such as intense skin lesions at mosquito bite sites and liver dysfunction, in patients with CAEBV and SHMB. Although the association between CAEBV and SHMB is also of inter- 


\section{OHSHIMA et al}

est, its mechanism has not yet been clarified.

In this study, we proposed a possible mechanism for the abnormal NK cell expansion in a patient with CAEBV and SHMB. Considering the high incidence of NK-GLPD in patients with CAEBV and SHMB, the present findings might contribute to the understanding of NK-GLPD pathogenesis.

Acknowledgements: We thank Prof. S. Nagata (Osaka University, Japan) for helpful discussions and generous donation of reagents (BB-2116; matrix metalloproteinase inhibitor, 4H9; biotinylated anti-Fas ligand, and recombinant soluble Fas ligand). We also thank Dr. H. Kanegane (Toyama Medical and Pharmacological University, Japan) for useful suggestions, and Ms. R. Ishida for secretarial assistance.

\section{References}

1) Galindo PA, Gomez E, Borja J, et al. Mosquito bite hypersensitivity. Allergol Immunopathol Medr 26: 251-254, 1998.

2) Mohri S, Kawashima Y, Uchigata Y, et al. A case of mosquito hypersensitivity terminating as malignant histiocytosis. J Dermatol 9: 437-443, 1982.

3) Ishihara S, Ohshima K, Tokura Y, et al. Hypersensitivity to mosquito bites conceals clonal lymphoproliferation of Epstein-Barr viral DNA-positive natural killer cells. Jpn J Cancer Res 88: 82-87, 1997.

4) Tokura Y, Tamura Y, Takigawa M, et al. Severe hypersensitivity to mosquito bites associated with natural killer cell lymphocytosis. Arch Dermatol 126: 362-368, 1990.

5) Tokura $Y$, Ishihara $S$, Ohshima $K$, et al. Severe mosquito bite hypersensitivity, natural killer cell leukaemia, latent or chronic active Epstein-Barr virus infection and hydroa Vacciniforme-like eruption. Br J Dermatol 138: 905-906, 1998 (letter).

6) Tsuge I, Morishima T, Morita M, Kimura H, Kuzushima K, Matsuoka H. Characterization of Epstein-Barr virus (EBV)-infected natural killer (NK) cell proliferation in patients with severe mosquito allergy; establishment of an IL-2-dependent NK-like cell line. Clin Exp Immunol 115: 385392, 1999.

7) ThomasTE, Sutherland HJ, Lansdorp PM. Specific binding and release of cells from beads using cleavable tetrameric antibody complexes. J Immunol Methods 120: 221-231, 1989.
8) Nomura S, Wills AJ, Edwards DR, Heath JK, Hogan BLM. Developmental expression of 2ar (osteopontin) and SPARC (osteonectin) RNA as revealed by in situ hybridization. J Cell Biol 106: 441-450, 1988.

9) Saito I, Servenius B, Compton T, Fox RI. Detection of Epstein-Barr virus DNA by polymerase chain reaction in blood and tissue biopsies from patients with Sjögren's syndrome. J Exp Med 169: 2191-2198, 1989.

10) Raab-Traub N, Flynn K. The structure of the termini of the Epstein-Barr virus as a marker of clonal cellular proliferation Cell 47: 883-889, 1986.

11) Kawa-Ha K, Ishihara $S$, Ninomiya $T$, et al. CD3-negative lymphoproliferative disease of granular lymphocytes containing Epstein-Barr viral DNA. J Clin Invest 84: 51-55, 1989.

12) Kikuta H, Taguchi $Y$, Tomizawa K, et al. Epstein-Barr virus genome-positive $\mathrm{T}$ lymphocyte in a boy with chronic active $\mathrm{EBV}$ infection associated with Kawasaki-like disease. Nature 333: 455-457, 1988.

13) Brooks L, Yao QY, Rickinson AB, Young LS. Epstein-Barr virus latent gene transcription in nasopharyngeal carcinoma cells: coexpression of EBNA 1, LMP 1, and LMP 2 transcripts. J Virol 66: 2689-2697, 1992.

14) Chen CL, Sadler RH, Walling DM, Su IJ, Hsieh HC, Raab-Traub N. Epstein-Barr virus (EBV) gene expression in EBV-positive peripheral Tcell lymphomas. J Virol 67: 6303-6308, 1993.

15) Pallesen G, Hamilton-Dutoit SJ, Zhou X. The association of Epstein-Barr virus (EBV) with T cell lymphoproliferations and Hodgkin's disease: two new developments in the EBV field. Adv Cancer Res 62: 179-239, 1993.

16) Kube D, Vockerodt M, Weber O, et al. Expression of Epstein-Barr virus nuclear antigen 1 is associated with enhanced expression of CD25 in Hodgkin cell line L428. J Virol 73: 1630-1636, 1999.

17) Kawa-Ha K, Franco E, Doi S, et al. Successful treatment of chronic active Epstein-Barr virus infection with recombinant interleukin-2. Lancet 1: 154,1987 (letter).

18) Henderson $S$, Rowe $M$, Gregory $C$, et al. Induction of bcl-2 expression by Epstein-Barr virus latent membrane protein 1 protects infected $B$ cells from programmed cell death. Cell 65: 1107-1115, 1991.

19) Walker PR, Saas P, Dietrich PY. Role of Ras ligand (CD95L) in immune escape: the tumor cell strikes back. J Immunol 158: 4521-4524, 1997.

20) Tierney RJ, Steven N, Young LS, Rickinson AB. Epstein-Barr virus latency in blood mononuclear cells: analysis of viral gene transcription during primary infection and in the carrier state. J Virol 68: 7374-7385, 1994.

21) Tanaka M, Suda T, Haze K, et al. Fas ligand in human serum. Nat Med 2: 317-322, 1996. 